Groups Geom. Dyn. 5 (2011), 495-496

DOI $10.4171 / \mathrm{GGD} / 192$
Groups, Geometry, and Dynamics

(C) European Mathematical Society

\title{
Erratum to "How to read the length of a braid from its curve diagram"
}

\author{
Groups Geom. Dyn. 5 (2011), 673-681
}

Tetsuya Ito and Bert Wiest

\begin{abstract}
Proposition 3.1 in [1] is incorrect. We give a counterexample, point out the mistake in the proof, but also propose a similar statement in the form of a conjecture. The main result of the paper (Theorem 2.1) is unaffected.
\end{abstract}

Mathematics Subject Classification (2010). 20F36, 20 F10.

Keywords. Braid group, Garside group, curve diagram.

In the article [1], a certain labelling of the curve diagram of a braid by integer numbers was defined, called the winding number labelling. It was shown (Theorem 2.1) that the maximal and minimal label occurring in the curve diagram of a braid $\beta$ is equal to the supremum and infimum of $\beta$, in the sense of Garside theory.

It was then claimed (Proposition 3.1) that the occurrences of the maximal and minimal labels in the diagram are rare, in the sense that there are at most $n-1$ of each, where $n$ denotes the number of strands. The purported proof of this result passed through a technical statement (Proposition 3.1') which asserted, among other things, that there could never be two parallel arc segments (each segment having its endpoints in two successive vertical tangencies), both carrying the maximal label.

Proposition $3.1^{\prime}$ is incorrect, and Figure 1 (a) below contains the curve diagram of a braid $\beta_{1}$ in $B_{6}$ which is a counterexample (here braids act on the right). In a similar manner, one can construct curve diagrams of braids in $B_{2 k+2}$ where all labels belong to $\{-1,0,1\}$, and where there are $k$ parallel arcs labelled 1 .

Starting from the braid $\beta_{1}$ we can even construct a counterexample to Proposition 3.1 of [1]: the curve diagram of the braid $\beta=\beta_{1} \beta_{2} \in B_{6}$, where $\beta_{2}=$ $\sigma_{2}^{3} \sigma_{4}^{3} \sigma_{1}^{2} \sigma_{3}^{2} \sigma_{5}^{2}$, has six occurrences of the maximal label 3, as shown in Figure $1(\mathrm{~b})$.

Yet, we believe that the slogan "maximally labelled arcs are rare" is philosophically true. To be more precise, let us use the following notation: the curve diagram of a braid $\beta$ consists of the $\operatorname{arcs} E_{1} \cdot \beta, \ldots, E_{n-1} \cdot \beta$, where $E_{i}$ is the straight arc between the $i$ th and $(i+1)$ st puncture. 
(a)

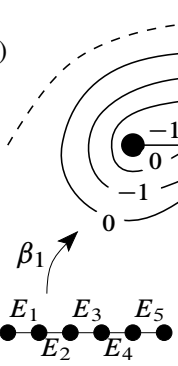

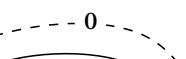
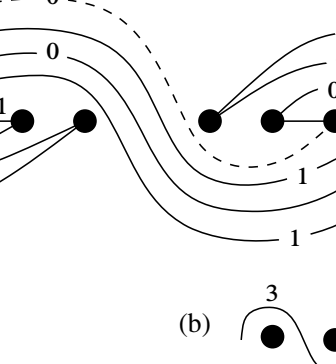

0
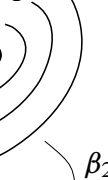

$\beta_{2}$
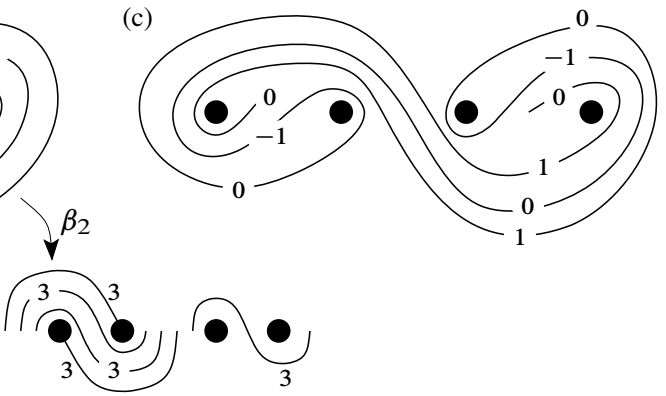

Figure 1. (a) The curve diagram of the braid

$$
\beta_{1}=\sigma_{3}^{-1} \sigma_{1}^{-1} \sigma_{2}^{-1} \sigma_{1}^{-1} \sigma_{5}^{-1} \sigma_{4}^{-1} \sigma_{5}^{-1} \cdot \sigma_{3} \sigma_{2} \sigma_{4} \sigma_{3} \sigma_{1} \sigma_{2} \sigma_{5} \sigma_{4} \sigma_{3} .
$$

There are two parallel arcs labelled 1. (b) The maximally labelled arcs of the curve diagram of the braid $\beta=\beta_{1} \beta_{2}$, with $\beta_{2}=\sigma_{2}^{3} \sigma_{4}^{3} \sigma_{1}^{2} \sigma_{3}^{2} \sigma_{5}^{2}$. (c) The error of the paper [1] occurred in the proof of Proposition 3.1', where the arc shown in (c) was claimed not to exist.

Conjecture 1. For any braid $\beta \in B_{n}$ and any $i \in\{1, \ldots, n-1\}$, the arc $E_{i} \cdot \beta$ of the curve diagram, equipped with the winding number labelling, contains no two parallel arc segments which both carry the maximal label.

For instance, this conjecture asserts that the arc in Figure 1 (c) cannot occur in a curve diagram with maximal label 1 . This conjecture, if true, would have a similar effect as the wrong Proposition 3.1: it would place a bound depending only on $n$ on the number of arcs carrying the maximal winding number labelling.

\section{References}

[1] B. Wiest, How to read the length of a braid from its curve diagram. Groups Geom. Dyn. 5 (2011), 673-681. Zbl 06010075 MR 2813531

Received January 3, 2012; revised February 14, 2012

T. Ito, Research Institute for Mathematical Sciences, Kyoto University, Sakyo-ku, Kyoto, 606-8502, Japan

E-mail: tetitoh@kurims.kyoto-u.ac.jp

B. Wiest, IRMAR, UMR 6625 du CNRS, Université de Rennes 1, Campus de Beaulieu, 35042 Rennes Cedex, France

E-mail: bertold.wiest@univ-rennes1.fr 